\title{
Análisis de la agencia epistémica de los estudiantes en un contexto argumentativo
}

\author{
Tatiana Costa Ramos (iD, Paula Cardoso Cristina Mendonça (D), \\ Nilmara Braga Mozzer (D) \\ Universidade Federal de Ouro Preto, Instituto de Ciências Exatas e Biológicas, Programa de Pós-graduação \\ em Educação, OuroPreto,MG,Brasil.tatianaquimica@outlook.com,paulamendonca@ufop.edu.br, \\ nilmara@ufop.edu.br
}

[Recibido: 18 junio 2020. Revisado: 30 junio 2020. Aceptado: 4 febrero 2021]

\begin{abstract}
Resumen: En el presente trabajo analizamos el desarrollo de la autonomía y la responsabilidad por parte de los estudiantes al observar su actuación como agentes epistémicos en un contexto argumentativo de evaluación crítica de analogías. Esta investigación se basa en el supuesto de un enfoque cualitativo y asume el carácter de investigación basada en diseño. Con el objetivo de alcanzar nuestro objetivo se elaboró una secuencia didáctica y su desarrollo se llevó a cabo en un aula de Química compuesta por 35 estudiantes de 15 y 16 años. Todos los momentos de la recogida de datos fueron grabados en video y audio. El análisis señala que cuatro aspectos fueron fundamentales para la actuación de los estudiantes como agentes epistémicos, a saber: (i) la actuación de la docente con acciones dirigidas a apoyar la implicación de los estudiantes en el discurso y la legitimación de sus ideas, (ii) la igualdad moderada permitida entre los miembros del aula, (iii) el contexto de resolución de problemas, que favoreció la responsabilidad de los estudiantes de buscar soluciones coherentes y fundamentadas y (iv) el hecho de que estuvieran trabajando con analogías autorales como herramientas epistémicas. Por lo tanto, defendemos que la enseñanza de Química debe realizarse de tal manera que proporcione la equidad de la autoridad intelectual, considerando la implicación de los estudiantes como agentes epistémicos a partir del uso de herramientas epistémicas, como las analogías. Para ello, se debe alentar a los estudiantes a evaluar críticamente esas herramientas, a partir de contextos argumentativos de resolución de problemas en los cuales el docente considera fundamentalmente las propuestas que ellos formulen.
\end{abstract}

Palabras clave: Argumentación; Agente epistémico; Analogías

\section{Analysis of the epistemic agency of students in an argumentative context}

Abstract: In this paper, we analyze the development of autonomy and responsibility of students when observing their performance as epistemic agents in an argumentative context of critical evaluation of analogies. This research is based on the assumption of a qualitative approach and assumes the character of design-based research. In order to achieve our objective, a didactic sequence was elaborated and its development was carried out in chemistry classes composed of 35 students aged between 15 and 16 years old. All data were recorded in both video and audio. The analysis indicates that four aspects were fundamental to the actions of students as epistemic agents, these being: (i) the interaction of the teacher seeking to support the participation of students in the discourse and legitimization of their ideas, (ii) allowed tempered equality among class members (iii) the context of problem-solving, which favored the autonomy of students in seeking coherent and knowledge-based solutions and (iv) working with authorial analogies as epistemic tools. In this manner, we advocate the teaching of Chemistry should be driven to give opportunity to the equality of intellectual authority, in particular considering the involvement of students as epistemic agents using epistemic tools, such as analogies. Aiming this, students need to be encouraged to critically evaluate these tools, based on argumentative problem-solving contexts in which their proposals are fundamentally considered by the teacher.

Keywords: Argumentation; Epistemic Authority; Analogy

Para citar este artículo: Ramos, T. C., Mendonça, P. C. C. y Mozzer, N. B. (2021) Análisis de la agencia epistémica de los estudiantes en un contexto argumentativo. Revista Eureka sobre Enseñanza y Divulgación de las Ciencias 18(2), 2102. doi: 10.25267/Rev_Eureka_ensen_divulg_cienc.2021.v18.i2.2102 


\section{Introducción}

El campo de la Didáctica de las Ciencias viene enfatizado la inserción de prácticas científicas en la enseñanza de ciencias para que los estudiantes puedan, además de desarrollar el conocimiento conceptual, convertirse en participantes legítimos en las dimensiones sociales y epistémicas que caracterizan las prácticas de la ciencia (Christodoulou y Osborne, 2014; Osborne, 2016; Sasseron y Duschl, 2016).

La implicación de los estudiantes en las prácticas científicas puede proporcionar una comprensión de la dimensión epistémica de la ciencia, que incluye comprender 'cómo sabemos lo que sabemos', es decir, sobre cómo los procesos de producción del conocimiento justifican nuestra creencia en ciertas afirmaciones de la ciencia (Jiménez-Aleixandre, 2010; Osborne, 2016). La participación en las prácticas científicas también incluye que los estudiantes puedan experimentar la dimensión social de la ciencia, ya que tiene como objetivo acercar la cultura del aula a la cultura científica, en la que el conocimiento se produce y negocia socialmente a partir de las comunidades de práctica (Nascimento y Sasseron, 2019). Por lo tanto, podemos afirmar que la experiencia de los estudiantes en las prácticas científicas puede contribuir a que comprendan cómo se producen los debates de los conceptos e ideas en la comunidad científica y qué es lo que cuenta en la toma de decisiones de esa comunidad, a partir de la construcción social del conocimiento (Sasseron, 2018).

En este sentido, las investigaciones en el área (Kelly, 2008; Osborne, 2016; Sasseron, 2018; Stroupe, 2014) también defienden la proyección de ambientes de enseñanza en los que el aprendizaje se produce a partir de interacciones discursivas, buscando la construcción y la evaluación social del conocimiento, en lugar de enfatizar en exceso la autoridad epistémica del docente. Consideramos que el profesor, como portavoz del discurso de la ciencia, tiene el importante papel de otorgar un estatuto epistémico al conocimiento escolar (Chiaro y Leitão, 2005). Sin embargo, este papel no inviabiliza que se les conceda autoridad y responsabilidad a los estudiantes en la negociación del conocimiento durante la enseñanza y el aprendizaje de Ciencias (Engle y Conant, 2002).

Esto se relaciona con lo que Stroupe (2014) denomina participación legítima de los estudiantes en las prácticas científicas, cuando se permite que asuman el papel de agentes epistémicos en el contexto de la enseñanza y el aprendizaje de Ciencias. Para este autor, los estudiantes son llamados agentes epistémicos cuando desarrollan autonomía y responsabilidad para construir el conocimiento y las prácticas de la comunidad del aula. El autor agrega que en las aulas donde se alienta el desarrollo del rol de agentes epistémicos de los estudiantes, esta comunidad considera centrales las ideas de estos estudiantes. $\mathrm{Al}$ asumir ese rol, los estudiantes aprenden a reconocer que sus ideas tienen valor y que a cada uno de ellos se le permite (y se espera) trabajarlas y negociarlas en el ámbito social (Stroupe, 2014).

El desarrollo de ambientes argumentativos en la enseñanza puede favorecer la actuación de los estudiantes como agentes epistémicos en la medida en que se brinda oportunidades para interacciones buscando la evaluación, la crítica, la refutación y la legitimación de ideas a partir de evidencias (Christodoulou y Osborne, 2014; Ferraz y Sasseron, 2017; Jiménez-Aleixandre, 2010). Sin embargo, estos ambientes se han promovido en las aulas con un mayor énfasis en la construcción del conocimiento por parte de los estudiantes, más que en los procesos de crítica, evaluación y negociación social de este conocimiento (Osborne, MacPherson, Patterson, y Szu, 2012).

En este sentido, los estudios sobre argumentación han demostrado que los estudiantes tienen dificultades para criticar y evaluar ideas entre sus compañeros porque no ven las evidencias

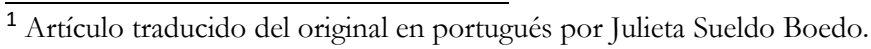


como centrales en las legitimaciones de las explicaciones, no reconocen las lagunas en los argumentos y en las explicaciones propuestas por otros o piensan que este rol pertenece solo al profesor (Jiménez-Aleixandre, Bugallo Rodríguez, y Duschl, 2000). Trabajos como los de Chiaro y Leitão (2005) y McNeill, González-Howard y Suzanna (2017) ponen en evidencia que a menudo los argumentos se centran en las interacciones profesor-estudiante debido a la falta de autonomía de los estudiantes y a que se centran en la autoridad epistémica del docente, considerado por ellos el único agente capaz de proponer, evaluar y legitimar ideas en el ámbito social de esa comunidad.

Por lo tanto, es relevante analizar cómo los estudiantes desarrollan autonomía y responsabilidad y se convierten en agentes epistémicos en ambientes argumentativos, con el objetivo de dilucidar aspectos que pueden contribuir a que asuman este rol en el contexto de la enseñanza de Ciencias.

\section{Prácticas epistémicas, argumentación y agentes epistémicos en la enseñanza de ciencias}

Las prácticas epistémicas son las formas con las que los miembros de una comunidad producen, evaluan, comunican y legitiman el conocimiento a partir de las múltiples interacciones sociales que se establecen (Kelly, 2008; Kelly y Duschl, 2002). El énfasis en trabajar con estas prácticas en la enseñanza de Ciencias se debe al hecho de que posibilitan el desarrollo de comunidades de prácticas que brindan oportunidades para la construcción y evaluación social del conocimiento (Lave y Wenger, 1991).

En este sentido, el desarrollo de comunidades de prácticas en el aula se vincula a la visión del trabajo científico como construido socialmente, en las que los estudiantes tienen la oportunidad de actuar como agentes epistémicos, es decir, tienen autonomía para exponer, evaluar y criticar ideas en las discusiones, siendo sus propias ideas fundamentales para la negociación del conocimiento (Stroupe, 2014). Las comunidades de práctica en el aula hacen posible que los estudiantes construyan nuevas identidades, ya que sienten que son una parte activa de ese contexto (Lave, 1996).

Por esta razón, en los últimos años los trabajos sobre prácticas epistémicas en la Didáctica de las Ciencias han ganado importancia (Jiménez-Aleixandre y Crujeiras, 2017; Kelly, 2008; Osborne, 2016; Sasseron y Duschl, 2016). Las investigaciones han señalado una gama de prácticas epistémicas como las que se espera que los estudiantes activen en contextos de enseñanza en los que se requiere la participación en los procesos de justificación del pensamiento, lo que les permite desarrollar una autonomía intelectual y discursiva. Algunos ejemplos de estas prácticas son: predicción de datos en una situación de investigación (buscando interacciones de proposición de conocimiento); construcción de argumentos basados en evidencias (buscando interacciones para la comunicación del conocimiento); evaluación sobre los méritos de una afirmación o evidencia (buscando interacciones para la evaluación del conocimiento); construcción de consenso sobre la mejor explicación para ese momento de la investigación (buscando interacciones para la legitimación del conocimiento) (Kelly y Licona, 2018).

Podemos afirmar que existe un fuerte vínculo entre la argumentación y la promoción de prácticas epistémicas (Christodoulou y Osborne, 2014). Esto porque la argumentación es un proceso a partir del cual los individuos pueden reconocer la plausibilidad de las soluciones a un problema o situación, ante la ponderación de los argumentos, que se justifican por medio de informaciones (Baker, 2009). Por eso, en ambientes argumentativos pueden ocurrir distintas prácticas epistémicas, como las que se destinan a evaluar los méritos de las 
explicaciones rivales para resolver el problema y construir un consenso sobre la mejor solución.-

Desde esta perspectiva, las estrategias dirigidas a promover ambientes argumentativos en la enseñanza de Ciencias pueden proporcionar un contexto favorable para el desarrollo de la autonomía intelectual y discursiva por parte de los estudiantes, ya que les permite implicarse en diferentes prácticas epistémicas. Esto se debe a que, como lo demuestran Silva et al. (2018), la implicación de los estudiantes en una mayor diversidad de prácticas epistémicas puede contribuir al desarrollo de su autonomía. Las autoras observaron que esa autonomía para participar en el discurso en el aula se produjo cuando los estudiantes se involucraron en prácticas tales como: proponer hipótesis para explicar los resultados de un experimento; evaluar las explicaciones propuestas; analizar y evaluar datos para la selección de evidencias.

Sin embargo, como señalan Mortimer y Araújo (2014), Stroupe (2014), McNeill et al. (2017) y Sasseron (2018), el desarrollo de la autonomía de los estudiantes depende de cómo el docente hace uso del discurso para involucrarlos en las interacciones en el aula. Mortimer y Araújo (2014) subrayan que en el aula tradicional los docentes no les conceden a los estudiantes autoridad para trabajar los problemas abordados. Como destacó Sasseron (2018), en un ambiente social de aprendizaje, el docente puede usar su autoridad epistémica para favorecer un escenario para el desarrollo de la autonomía de los estudiantes, ya que valora y fomenta la exposición de ideas por parte de ellos, así como alienta el trabajo con las prácticas de la ciencia. En este sentido, Stroupe (2014) enfatiza que los estudiantes tienen que ser vistos como sujetos que pueden poner de manifiesto sus conocimientos y sus puntos de vista y que estos sean debidamente considerados por el docente para su implicación en las discusiones y los conocimientos en debate.

Esta visión está respaldada por datos del trabajo de McNeill et al. (2017). Los autores analizaron la variación en las instrucciones de diversos docentes al promover la argumentación en el aula. Señalaron que algunos profesores les daban a los estudiantes un espacio restringido para hablar, lo que limitaba sus oportunidades de actuar como agentes epistémicos. Esto sucedía, especialmente, debido a que los profesores buscan "respuestas correctas", situaciones en las que solo se tomaban en cuenta los discursos de los estudiantes que coincidían con lo que los docentes querían escuchar. Por otro lado, otros docentes formulaban cuestionamientos que ayudaban a los estudiantes a involucrarse en la discusión, brindando oportunidades para su participación en interacciones para proponer y comunicar ideas.

Es importante destacar que, como lo expresaron Jiménez-Aleixandre et al. (2000), la promoción de la argumentación en la enseñanza también se ve influenciada por la visión epistemológica del profesor. En este caso, la relación que el docente establece entre la argumentación en la enseñanza de Ciencias en la escuela y la práctica argumentativa en el contexto de la ciencia influye en cómo ve el rol autoral del estudiante en la elaboración y evaluación del conocimiento, así como su propio rol en la comunidad de práctica.

En el trabajo de Chiaro y Leitão (2005), las autoras mostraron que algunas veces los estudiantes se implican en interacciones para la comunicación y la producción de ideas con sus compañeros, pero en procesos de construcción del conocimiento es menos probable que se perciban a sí mismos y a sus compañeros como agentes epistémicos en posiciones de evaluar, criticar y legitimar conclusiones establecidas por alguno de estos participantes sobre el tema en discusión. En estos procesos, observaron que fueron las acciones discursivas del profesor lo que le confirieron un status epistémico al discurso de los estudiantes y contribuyeron con la construcción y legitimación del conocimiento. 
En línea con la opinión de los demás autores, Christodoulou y Osborne (2014), al investigar en contextos argumentativos en la enseñanza de Ciencias, observaron que los docentes prestan demasiada atención a las interacciones destinadas a proponer y comunicar ideas por parte de los estudiantes. Por eso, hay que hacer una aclaración sobre la implicación del trabajo antes mencionado, en lo que respecta a la necesidad de que el discurso del docente también brinde a los estudiantes la oportunidad de implicarse en interacciones que se destinen a criticar y evaluar las declaraciones. En este trabajo pretendemos presentar una estrategia de enseñanza alineada con este objetivo y con el de promover la autonomía de los estudiantes en el contexto de la enseñanza de Ciencias.

\section{Las analogías y la enseñanza de química}

La enseñanza de Química involucra temas con conceptos abstractos como modelos atómicos, enlaces químicos y equilibrio químico, que son algunos ejemplos de temas que los estudiantes consideran "difíciles". Por eso, es común que los docentes y autores de materiales de instrucción usen analogías para favorecer la comprensión de estos temas por parte de los estudiantes (Aragón y Oliva, 2013; Bernardino, Rodrigues, y Bellini, 2013; Cascales, Blanco, y Pérez, 2018).

Gentner (1989) define las analogías como comparaciones en las que un concepto o una situación familiar (dominio análogo) comparte relaciones de similitud con el concepto o situación desconocida o poco familiar (dominio objetivo). En este sentido, por medio de analogías, se pueden reconocer similitudes entre objetos, fenómenos, estructuras, etc., que pueden ser físicamente distintos entre sí, pero cuyas entidades constituyentes juegan roles similares en las estructuras de los dominios comparados.

Cuando se usan analogías para ayudar a los estudiantes a comprender conceptos, estas se denominan modelo de enseñanza, es decir, modelos creados con el objetivo de ayudar a los estudiantes a comprender aspectos del concepto que se les va a enseñar (Justi, 2006). Cuando se usan las analogías como modelos de enseñanza le dan a ese contexto un objeto, una situación o un fenómeno que le es familiar al estudiante, con el objetivo de brindarle apoyo para que pueda elaborar ideas coherentes del conocimiento científico.

A pesar de la intención de que se usen como modelos de enseñanza, algunas analogías no siempre son favorables para la comprensión de los estudiantes sobre el objeto de estudio. Este es el caso de la analogía entre el modelo atómico de Thomson y el "pudín de pasas", cuyas inconsistencias derivadas de su uso en la enseñanza del modelo han sido destacadas en varios trabajos (Blanco, Alemany, y Torregrosa, 2013; Lopes y Marques, 2010; Ramos y Mozzer, 2018; Rosa, Cótica y Pereira, 2016).

La analogía del "pudín de pasas" se popularizó como un modelo de enseñanza para facilitar la comprensión del modelo atómico de Thomson. En esta analogía, como se ha mencionado, el análogo es el "pudín de pasas" de origen inglés (o "plum pudding 2 "), y el objetivo es el modelo atómico de Thomson. Esta analogía, cuando se discute adecuadamente, puede facilitar la comprensión de un solo aspecto del modelo atómico de Thomson: la distribución homogénea de cargas. Por lo tanto, el uso de la analogía del "pudín de pasas" como un modelo de enseñanza es muy limitado.

Distintas investigaciones discuten sobre esta analogía en el contexto de la enseñanza de Ciencias (Blanco, et al. 2012; Lopes y Martins, 2009; Ramos y Mozzer, 2018). Blanco et al. (2012) evaluaron de qué manera los libros de texto incorporan características epistemológicas del conocimiento en la enseñanza de los modelos de Thomson y Rutherford y se dieron

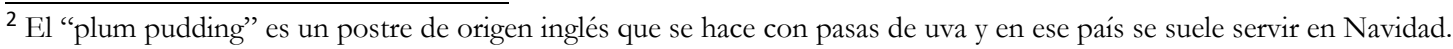


cuenta de que la discusión basada en la analogía con el "pudín de pasas" puede desencadenar una visión simplista del átomo. Además, los autores señalan que las ideas de Thomson difieren de las que se presentan en los libros a partir de la discusión de la analogía. Ramos y Mozzer (2018) investigaron la presentación de esta analogía a los estudiantes a partir del modelo de instrucción "Teaching With Analogies" (TWA) propuesto por el autor Glynn (2007), y también observaron una comprensión limitada de los estudiantes sobre el concepto a partir de la analogía.

Lopes y Martins (2009) discutieron las características originales de las ideas de Thomson sobre el átomo, con el objetivo de discutir las relaciones de similitud que se han establecido entre las ideas de Thomson y el análogo "pudín de pasas". Las investigaciones citadas demuestran que la analogía con el "pudín de pasas" no facilita la comprensión de los estudiantes del modelo atómico de Thomson y algunas, incluso, señalan la inconsistencia de esta analogía para representar las ideas de Thomson, en particular, la del dinamismo que se refiere al movimiento constante de los electrones en el modelo propuesto a partir de un "pudín de pasas" estático.

Aliado a esto, investigaciones como la de Rosa et al. (2016) y Ramos y Mozzer (2018) destacan la falta de familiaridad de algunos estudiantes con el análogo "pudín de pasas", ya que el análogo es un pudín de origen inglés. Por lo tanto, esta problemática conlleva una cuestión cultural y, en estos casos, la analogía establecida no cumple su función como modelo de enseñanza, ya que, para ello, es esencial que la analogía sea familiar para los estudiantes (Duit, 1991; Thiele y Treagust, 1994).

Teniendo en cuenta estos aspectos, compartimos las ideas de Mozzer y Justi (2015) y Cascales, et al. (2018) sobre el potencial de crear analogías por parte de los propios estudiantes, permitiéndoles elaborar, expresar y evaluar sus comparaciones. Esto se debe a que, al crear sus propias analogías, los estudiantes tendrán que elegir análogos que les sean familiares, por lo que, asimismo, se resuelven los problemas con la falta de comprensión del análogo. Además, como lo destacan Cascales et al. (2018), este proceso puede favorecer la autonomía y la participación de los estudiantes en el aula.

Asimismo, creemos que la elaboración, expresión y evaluación crítica de comparaciones dentro de un ambiente argumentativo de enseñanza favorece la participación de los estudiantes en las interacciones y les permite posicionarse, proponer y evaluar las comparaciones que surgen en el ámbito social del aula. Por lo tanto, en el presente trabajo analizamos la actuación de los estudiantes como agentes epistémicos en un contexto argumentativo en el que se les alentó a analizar críticamente la analogía con el "pudín de pasas" en un proceso de elección entre las analogías creadas por ellos mismos y las presentadas por el libro de texto.

Es importante destacar que, aunque otros estudios ya han desarrollado análisis sobre la comprensión de los estudiantes de la analogía con el "pudín de pasas" y el modelo atómico de Thomson, nuestro trabajo representa un avance en estas investigaciones, ya que se centra en el análisis de la promoción de la autonomía de los estudiantes al evaluar críticamente esta analogía en un ambiente argumentativo de la enseñanza de Química.

\section{Metodología}

El abordaje metodológico adoptado en el presente trabajo se basa en los principios teóricometodológicos de la investigación cualitativa en educación (Lüdke y André, 2015). Más específicamente, nuestro trabajo asume el carácter de investigación basada en diseño (Kneubil y Pietrocola, 2017; Collins, José y Bielaczyc, 2004), ya que se alinea con el objetivo de llevar al aula procesos y metodologías orientados a perfeccionar la enseñanza y el aprendizaje. En 
nuestra investigación, desarrollamos una secuencia didáctica (SD) con el objetivo de favorecer la agencia epistémica de los estudiantes y promover interacciones argumentativas en un aula de Química.

La recogida de datos tuvo lugar en un aula de Química de una escuela pública situada en Brasil. La clase estaba compuesta de 35 estudiantes, cuya edad estaba entre los 15 y los 16 años. Se seleccionó el nivel de enseñanza en base a la elección de trabajar con la analogía entre el "pudín de pasas" y el modelo atómico de Thomson. La secuencia didáctica (SD) se desarrolló para enseñar los modelos atómicos de Dalton y Thomson (Ramos, Mendonça y Mozzer, 2016) y duró 4 (cuatro) semanas y un total de 10 (diez) clases de Química, de 50 (cincuenta) minutos cada una, cuyo seguimiento las investigadoras llevaron a cabo durante ese período. Inicialmente, la SD fue elaborada por las autoras de este manuscrito y, posteriormente, se realizaron dos reuniones con la docente colaboradora de esta investigación con el fin de discutir la SD con ella y brindarle oportunidades de modificar las actividades de acuerdo con sus sugerencias, en consonancia con los supuestos de la investigación basada en diseño. Por lo tanto, antes del desarrollo de la SD en el aula, las actividades sufrieron pequeños ajustes de acuerdo con estas sugerencias.

La enseñanza que solían experimentar los estudiantes en esa clase se basaba en la presentación de los conceptos por parte del docente y en su "recepción" por parte de los estudiantes. Por eso, no estaban acostumbrados a trabajar con prácticas científicas destinadas a proponer, comunicar y evaluar ideas. De esta forma, buscando favorecer la comunicación entre los estudiantes y posibilitar oportunidades para el desarrollo de un ambiente argumentativo, la clase se organizó en grupos de cuatro estudiantes en todos los momentos de la recogida de datos, que se grabaron en video y audio.

Asimismo, también como premisa de la investigación basada en diseño (Kneubil y Pietrocola, 2017), la investigadora ejerció un papel activo en el escenario de esta investigación, actuando como observadora participante (Cohen et al., (2011), aclarando las dudas de los estudiantes y ayudándoles a presentar sus ideas, con el objetivo de promover su implicación en interacciones argumentativas.

La secuencia didáctica que se desarrolló buscaba promover un ambiente argumentativo a partir de la creación y el análisis crítico de analogías por parte de los estudiantes sobre los modelos atómicos de Dalton y Thomson. En el presente trabajo, la atención se centra en los momentos de esta secuencia que se destinan a que los estudiantes elijan entre las analogías que crearon para representar las ideas relacionadas con el modelo atómico de Thomson y con el "pudín de pasas" presentado por el libro de texto. Elaboramos tablas de análisis de las comparaciones para explicitar las correspondencias establecidas entre las similitudes identificadas entre los dominios análogo y objetivo y las limitaciones de las analogías. En estas tablas, las flechas bidireccionales enteras indican una relación de similitud entre los dos dominios y las flechas bidireccionales discontinuas señalan que la similitud entre los dominios es un atributo de objeto (propiedades descriptivas). El símbolo de distinto, por otro lado, indica una limitación de esa comparación.

El recorte de análisis de los momentos de "elección entre analogías" de los estudiantes se justifica por nuestro objetivo de analizar su rol como agentes epistémicos. Partimos de la hipótesis de que este rol sería más expresivo en el momento en que los estudiantes se encontraban en un proceso de evaluación crítica de la analogía que ellos habían propuesto y la que suele presentar el libro de texto. Esto se ha hecho así porque, en ese momento, tendrían que evaluar una información que generalmente se considera un argumento de autoridad y, por lo tanto, se acepta sin una evaluación crítica. Los argumentos de autoridad son aquellos en los que la información proviene de fuentes que se consideran de confianza (como de los expertos, 
por ejemplo). Se aceptan sin presentar evidencias ni cuestionamientos, debido a la credibilidad que se le da al informante (Jiménez-Aleixandre, 2010).

Para el análisis de los datos la investigadora transcribió los momentos en que hubo interacción discursiva entre los miembros del aula. Se utilizaron puntos suspensivos entre paréntesis para expresar los momentos de pausa durante el diálogo. Los turnos al hablar correspondientes a las interacciones argumentativas establecidas se enumeraron de acuerdo con su principio y fin, es decir, el turno número 1 indica el comienzo de la interacción argumentativa.

A partir de la transcripción de los datos, seleccionamos, de acuerdo con los supuestos de Baker (2009), las interacciones argumentativas que ocurrieron en ese momento de la evaluación crítica de la analogía con el "pudín de pasas" y las analogías creadas por los estudiantes. Según Baker (2009), las interacciones argumentativas pueden entenderse como intentos de decidir sobre soluciones alternativas. Los argumentos son la información que se enuncia durante la resolución del problema y que alteran la aceptación de la solución.

\section{Resultados y discusiones}

Durante la discusión sobre la analogía con el "pudín de pasas" se observaron dos momentos en los que ocurrieron interacciones argumentativas. A continuación, presentamos cada una en orden cronológico.

La interacción argumentativa presentada en la tabla 1 ocurrió cuando se les pidió a los estudiantes que eligieran entre la analogía que ellos mismos habían creado y la analogía con el "pudín de pasas" como la que mejor expresaba el modelo atómico de Thomson. En ese momento, la docente presentó a los estudiantes la relación entre el "pudín de pasas" y el modelo atómico y les informó que esa analogía se encontraba en el libro de texto en el que estudiaban.

Tabla 1. Interacción argumentativa 1

\begin{tabular}{|c|c|c|}
\hline $\begin{array}{l}\text { Turnos de } \\
\text { habla }\end{array}$ & Transcripción & Aclaraciones \\
\hline 1 & $\begin{array}{l}\text { [Profesora]: Ahora vamos a pensar en la pregunta cuatro. } \\
\text { ¿Cuál les parece que sería la mejor comparación para } \\
\text { expresar el átomo de Thomson? }\end{array}$ & $\begin{array}{l}\text { Problematización: elección entre la } \\
\text { analogía sugerida por los estudiantes } \\
\text { y la analogía del "pudín de pasas". }\end{array}$ \\
\hline 2 & [Carlos]: La del pudín es mejor que la nuestra, sí... & $\begin{array}{l}\text { El estudiante está a favor de la } \\
\text { analogía con el "pudín de pasas". }\end{array}$ \\
\hline 3 & [Profesora]: ¿Por qué? & $\begin{array}{l}\text { La profesora solicita justificación para } \\
\text { el posicionamiento del estudiante. }\end{array}$ \\
\hline 4 & $\begin{array}{l}\text { João]: Ah, porque el pudín es más esférico, no es solo } \\
\text { redondo... (...) Y los electrones se esparcen más... }\end{array}$ & $\begin{array}{l}\text { El estudiante justifica y comunica su } \\
\text { posición. }\end{array}$ \\
\hline 5 & $\begin{array}{l}\text { [Arthur]: Y las pasas, bueno... Las pasas son como en el } \\
\text { átomo... En los anillos... }\end{array}$ & $\begin{array}{l}\text { El estudiante completa la idea de su } \\
\text { compañero. }\end{array}$ \\
\hline 6 & $\begin{array}{l}\text { [Profesora]: Ok. Pero espera, ¿estás diciendo que las pasas } \\
\text { están como en los anillos aqui [refiriéndose al dibujo de } \\
\text { "plum pudding"]? }\end{array}$ & $\begin{array}{l}\text { La profesora busca alentar a los } \\
\text { estudiantes a reflexionar sobre su } \\
\text { justificación. }\end{array}$ \\
\hline 7 & [Arthur]: Sí... & El estudiante confirma su idea. \\
\hline 8 & $\begin{array}{l}\text { Profesora]: ¿Pero aquí [refiriéndose al dibujo del } \\
\text { "plum pudding"] las pasas están en anillos? }\end{array}$ & $\begin{array}{l}\text { La profesora pregunta sobre la relación } \\
\text { de las pasas con los electrones, en un } \\
\text { intento de promover la reflexión del } \\
\text { estudiante sobre la incoherencia de este } \\
\text { aspecto de la analogía. }\end{array}$ \\
\hline 9 & {$[$ Arthur]: No... } & $\begin{array}{l}\text { El estudiante empieza a reflexionar } \\
\text { sobre la organización de los electrones } \\
\text { en el átomo. }\end{array}$ \\
\hline
\end{tabular}


Tabla 1. Continuación.

\begin{tabular}{|c|c|c|}
\hline $\begin{array}{l}\text { Turnos de } \\
\text { habla }\end{array}$ & Transcripción & Aclaraciones \\
\hline 10 & $\begin{array}{l}\text { Profesora]: Ok. Entonces sigamos. ¿Crees que lo explica } \\
\text { mejor porque aqui [refiriéndose a la fotografía de un } \\
\text { "pudín de pasas"] es más esférico? ¿Esto es una esfera? }\end{array}$ & $\begin{array}{l}\text { La profesora retoma la idea del } \\
\text { alumno y la explota a partir del } \\
\text { análisis del análogo "pudín de pasas" }\end{array}$ \\
\hline 11 & [Carlos]: No. & \multirow{3}{*}{$\begin{array}{l}\text { Los estudiantes se dan cuenta de que } \\
\text { la forma del pudín no es similar a la } \\
\text { forma del átomo. }\end{array}$} \\
\hline 12 & [João]: No. & \\
\hline 14 & [Carlos]: No, es ovalado... & \\
\hline 15 & $\begin{array}{l}\text { Profesora]: Ok. Y otra cosa, dijiste que las pasas están } \\
\text { más distribuidas y comparamos las pasas con los electrones. } \\
Y \text { en el caso del átomo, ¿cómo están organizados los } \\
\text { electrones? }\end{array}$ & $\begin{array}{l}\text { La profesora retoma la idea del } \\
\text { estudiante y la evalúa para fomentar } \\
\text { la reflexión sobre la organización de } \\
\text { los electrones en el átomo. }\end{array}$ \\
\hline 16 & [Carlos]: Están esparcidos... & \multirow{2}{*}{$\begin{array}{l}\text { Arthur confirma la idea propuesta } \\
\text { por Carlos expresando que los } \\
\text { electrones se encuentran de forma } \\
\text { desorganizada en el átomo. }\end{array}$} \\
\hline 17 & $\begin{array}{l}\text { [Arthur]: Tal como lo mostramos ese día, no hay un orden } \\
\text { correcto... }\end{array}$ & \\
\hline 18 & $\begin{array}{l}\text { [Profesora]: Ajá... Pero bicieron la comparación de ustedes } \\
\text { por los anillos de los electrones, ¿no? Porque Thomson } \\
\text { cuando describió el átomo, dijo que tendrían anillos invisibles } \\
\text { donde estarían los electrones. Entonces, ¿me están diciendo } \\
\text { que los electrones se explican mejor aquí [refiriéndose al } \\
\text { "plum pudding"] por la distribución? ¿Todos están de } \\
\text { acuerdo? }\end{array}$ & $\begin{array}{l}\text { La profesora está de acuerdo y no } \\
\text { invalida las ideas de los estudiantes, } \\
\text { pero utiliza la comparación creada } \\
\text { por ellos para interrogarlos y evaluar } \\
\text { las ideas en debate. }\end{array}$ \\
\hline 19 & [Carlos]: Sí, bue... & \multirow{2}{*}{$\begin{array}{l}\text { Los estudiantes confirman que el } \\
\text { análogo del "pudín de pasas" es } \\
\text { mejor para expresar sus ideas sobre el } \\
\text { átomo. }\end{array}$} \\
\hline 20 & [Caio]: Estoy de acuerdo. & \\
\hline 21 & $\begin{array}{l}\text { Profesora]: Ok. ¿Pero qué pueden decirme que explique } \\
\text { que esta analogía es mejor que la de ustedes? (...) Ok. } \\
\text { Volvamos a las características del átomo para que podamos } \\
\text { entenderlo mejor. Thomson dijo que el átomo sería esférico, } \\
\text { que esa esfera tendría carga positiva. Los electrones se } \\
\text { distribuirían dentro de la esfera y estos electrones se } \\
\text { distribuirian en anillos que giran en un movimiento } \\
\text { constante, dentro de ese anillo. Y estos anillos tienen una } \\
\text { carga negativa. ¿Y entonces piensan que este de aqui } \\
\text { [refiriéndose al análogo de "plum pudding"] logra } \\
\text { explicar mejor el átomo? }\end{array}$ & $\begin{array}{l}\text { La profesora solicita que los } \\
\text { estudiantes justifiquen su posición. } \\
\text { Luego retoma las características del } \\
\text { átomo en un intento de presentar } \\
\text { evidencias que puedan respaldar los } \\
\text { argumentos de los estudiantes. }\end{array}$ \\
\hline 22 & [Carlos]: Sí. & $\begin{array}{l}\text { El estudiante no logra formular un } \\
\text { argumento para elegir el análogo } \\
\text { "pudín de pasas". }\end{array}$ \\
\hline 23 & $\begin{array}{l}\text { Profesora]: Ok. Y luego pueden explicar el fenómeno del } \\
\text { experimento, cuando decimos que las cargas fueron } \\
\text { desplazadas, ¿pueden explicar este fenómeno a través de esta } \\
\text { analogía del pudín de pasas? }\end{array}$ & $\begin{array}{l}\text { La profesora reanuda los datos de un } \\
\text { experimento realizado por los } \\
\text { estudiantes en busca de su reflexión } \\
\text { sobre el movimiento de los } \\
\text { electrones. }\end{array}$ \\
\hline 24 & [Carlos]: ¿Qué? & $\begin{array}{l}\text { El estudiante no entiende lo que se } \\
\text { interroga. }\end{array}$ \\
\hline
\end{tabular}

\footnotetext{
${ }^{3}$ Este experimento se había llevado a cabo en clases anteriores durante el desarrollo de la SD, en las que se abordaba la electrificación de un trozo de lana por la fricción con un globo, con el objetivo de discutir el movimiento de las cargas en el átomo.
} 
Tabla 1. Continuación.

\begin{tabular}{|c|c|c|}
\hline $\begin{array}{l}\text { Turnos de } \\
\text { habla }\end{array}$ & Transcripción & Aclaraciones \\
\hline 25 & $\begin{array}{l}\text { [Profesora]: ¿Me pueden explicar lo que pasó? Cuando me } \\
\text { dijeron lo que sucedió alli en el experimento fue porque hubo } \\
\text { un desplazamiento de carga. Y luego el globo tenía una carga } \\
\text { positiva, por lo que bubo un movimiento de los electrones. } \\
\text { Entonces, ipueden explicarme este fenómeno que sucedió alli, } \\
\text { mirando esta comparación [refiriéndose a la analogía } \\
\text { entre el pudín de pasas y el átomo de Thomson]? } \\
\text { (...) Es decir, esta comparación [refiriéndose a la } \\
\text { analogía del pudín de pasas], ipuede explicar el } \\
\text { movimiento de los electrones? }\end{array}$ & $\begin{array}{l}\text { La profesora reformula las preguntas } \\
\text { que había hecho previamente en } \\
\text { busca de la comprensión de los } \\
\text { estudiantes. }\end{array}$ \\
\hline 26 & [Arthur]: Ah, no... iNo lo creo! & $\begin{array}{l}\text { El estudiante se da cuenta de que el } \\
\text { análogo "pudín de pasas" no tiene } \\
\text { correspondencia con el movimiento } \\
\text { de los electrones presentes en el } \\
\text { átomo. }\end{array}$ \\
\hline 27 & [Caio]: ¿Por qué? & $\begin{array}{l}\text { El estudiante busca una justificación } \\
\text { de la afirmación de su compañero. }\end{array}$ \\
\hline 28 & $\begin{array}{l}\text { [Carlos]: Sí... Creo que no... Las pasas están representando } \\
\text { los electrones, ¿no? O sea, pero no, no se mueven alli } \\
\text { dentro... }\end{array}$ & $\begin{array}{l}\text { El estudiante comunica su idea y } \\
\text { utiliza la información del átomo para } \\
\text { evaluar la analogía con el "pudín de } \\
\text { pasas". }\end{array}$ \\
\hline 29 & [Profesora]: De acuerdo, no se mueven. & $\begin{array}{l}\text { La profesora está de acuerdo y } \\
\text { legitima la propuesta del alumno. }\end{array}$ \\
\hline 30 & $\begin{array}{l}\text { [Carlos]: Sí, bue... Es todo masa aquí [señalando la } \\
\text { figura del análogo "plum pudding"]... Se queda } \\
\text { detenida allí.. }\end{array}$ & $\begin{array}{l}\text { El estudiante critica el análogo } \\
\text { "pudín de pasas". }\end{array}$ \\
\hline 31 & $\begin{array}{l}\text { [oão]: iAh! Y en nuestra comparación aquí, la rueda sigue } \\
\text { girando, incluso cuando se detiene... }\end{array}$ & $\begin{array}{l}\text { El estudiante se da cuenta de que el } \\
\text { análogo inventado por el grupo es } \\
\text { más coherente para explicar la } \\
\text { dinamicidad del átomo. }\end{array}$ \\
\hline 32 & $\begin{array}{l}\text { Profesora]: ¿Entonces la comparación de ustedes lo puede } \\
\text { explicar? }\end{array}$ & $\begin{array}{l}\text { La profesora retoma la idea del } \\
\text { estudiante João a partir del } \\
\text { cuestionamiento. }\end{array}$ \\
\hline 33 & [Carlos]: Sí, lo explica... & Carlos contesta sin fundamentar. \\
\hline 34 & [Profesora]: ¿Por qué? & $\begin{array}{l}\text { La profesora solicita una justificación } \\
\text { para la afirmación del estudiante. }\end{array}$ \\
\hline 35 & $\begin{array}{l}\text { [Arthur]: Porque en el nuestro, incluso parado, sigue mo- } \\
\text { viéndose, el anillo... }\end{array}$ & $\begin{array}{l}\text { El estudiante justifica la analogía } \\
\text { creada por el grupo. }\end{array}$ \\
\hline 36 & $\begin{array}{l}\text { Profesora]: Ajá, porque ese círculo que dijeron que sigue } \\
\text { girando, serian los anillos de los electrones... Entonces } \\
\text { piensan que si sigue en movimiento alli [refiriéndose al } \\
\text { movimiento del anillo de la rueda del automóvil], } \\
\text { eso puede explicar ese movimiento de electrones que ocurre en } \\
\text { el átomo, ¿verdad? }\end{array}$ & $\begin{array}{l}\text { La profesora reformula la respuesta } \\
\text { del estudiante, presenta las } \\
\text { correspondencias entre el análogo } \\
\text { creado por el grupo y el modelo } \\
\text { atómico de Thomson a partir de las } \\
\text { preguntas sobre el movimiento de los } \\
\text { electrones. }\end{array}$ \\
\hline 37 & [Carlos]: Si, bue... & $\begin{array}{l}\text { Confirma la explicación dada por la } \\
\text { profesora. }\end{array}$ \\
\hline
\end{tabular}


En la interacción argumentativa transcrita anteriormente, observamos que cada uno de los estudiantes, Carlos, Arthur y Caio, se encontraba en un conflicto personal para decidirse entre la analogía con el "pudín de pasas" y la rueda del automóvil, que ellos habían creado. Para la comprensión del lector, presentamos las correspondencias de la analogía del pudín de pasas en la tabla 2 y la rueda del automóvil en la tabla 3.

Tabla 2. Representación de las correspondencias y limitaciones de la comparación con el "plum pudding”

\begin{tabular}{|c|c|c|}
\hline $\begin{array}{c}\text { Plum pudding } \\
\text { (análogo) }\end{array}$ & Correspondencias & $\begin{array}{c}\text { Modelo atómico de Thomson } \\
\text { (objetivo) }\end{array}$ \\
\hline $\begin{array}{c}\text { Pasas distribuidas por toda la } \\
\text { extensión del pudín }\end{array}$ & & $\begin{array}{c}\text { Electrones distribuidos por toda la } \\
\text { extensión del átomo }\end{array}$ \\
\hline $\begin{array}{c}\text { Masa del pudín distribuida } \\
\text { uniformemente }\end{array}$ & $\begin{array}{c}\text { Distribución uniforme de la parte } \\
\text { positiva del átomo }\end{array}$ \\
\hline Estático & Dinámico \\
\hline Pasas distribuidas aleatoriamente & Electrones dispuestos en anillos \\
\hline
\end{tabular}

Tabla 3. Representación de las correspondencias de la comparación con la rueda del automóvil

\begin{tabular}{|c|c|c|}
\hline $\begin{array}{c}\text { Rueda del vehículo } \\
\text { (análogo) }\end{array}$ & Correspondencias & $\begin{array}{c}\text { Modelo atómico de Thomson } \\
\text { (objetivo) }\end{array}$ \\
\hline $\begin{array}{c}\text { Anillo giratorio dentro de la } \\
\text { circunferencia de la rueda }\end{array}$ & $\begin{array}{c}\text { Electrones girando en anillos dentro } \\
\text { de la esfera del átomo }\end{array}$ \\
\hline $\begin{array}{c}\text { Anillo giratorio en el interior de la } \\
\text { rueda independientemente del } \\
\text { movimiento de la rueda }\end{array}$ & & $\begin{array}{c}\text { Anillo girando independientemente } \\
\text { del movimiento de la esfera }\end{array}$ \\
\hline
\end{tabular}

En la interacción argumentativa 1 (tabla 1) podemos ver que, inicialmente, los estudiantes parecían aceptar la analogía con el "pudín de pasas" porque lo consideraban un argumento de autoridad o porque no tenían convicción para defender sus ideas personales. A partir de las explicaciones proporcionadas por los estudiantes (turno 4) es notable que tenían conocimiento sobre el formato achatado del pudín y esférico del átomo, pero negaban esta evidencia porque iba en contra de la elección de la analogía del "pudín de pasas". Por eso, declararon que, como el pudín era "más esférico", sería un análogo más coherente.

En el turno 5, cuando el estudiante declara que "Y las pasas, bueno... Las pasas son como en el átomo... En los anillos...", destaca una información coherente sobre los electrones que se organizan en anillos. Pero, una vez más, el estudiante no siente seguridad como para criticar la analogía con el "pudín de pasas" y la defiende con base en evidencias que pesan en contra de la analogía, porque las pasas en un pudín están dispuestas al azar y no se distribuyen en anillos. De esta manera, es notable que los estudiantes insisten en aceptar la analogía con el "pudín de pasas", aunque sea contradictoria con sus propias ideas sobre el átomo. Por eso, los estudiantes entraron en conflicto con las visiones coherentes que habían desarrollado sobre un átomo dinámico, esférico y con electrones organizados en anillos cuando se encontraron con un análogo presentado en el libro que no explotaba estas ideas e incluso negaba algunas de ellas. En este caso, los estudiantes parecían realizar un ejercicio cognitivo de buscar fundamentos/información (turnos 27, 28) para decidir qué analogía elegir.

\footnotetext{
${ }^{4}$ Aunque consideramos que las relaciones establecidas por los estudiantes en la analogía entre la rueda del automóvil y el átomo de Thomson son válidas, sabemos que no son coherentes con el funcionamiento de la rueda del automóvil, ya que todo el sistema se detiene (excepto el motor) cuando se activa el freno.

5 Ídem nota anterior.
} 
Nuestros datos se muestran consistentes con las evidencias mostradas en el trabajo de Crujeiras (2014), que presenta que las acciones del docente con el objetivo de solicitar justificación promueven interacciones para proponer y comunicar ideas por parte de los estudiantes. Al igual que en el trabajo de esta autora, observamos que los estudiantes se implicaron en prácticas de comunicación de ideas (turnos 4 y 28) a medida que la docente les solicitaba las justificaciones (turnos 3 y 34 ) de sus posiciones. Esto señala que los estudiantes no se sentían seguros ni tampoco tenían convicción en sus ideas como para proponer o comunicar ideas sin la intervención de la docente.

Podemos notar que el auxilio de la docente (turnos $6,8,10,15)$ durante la interacción fue fundamental para que los estudiantes reflexionaran sobre sus proposiciones y argumentos. A partir de los interrogantes (turnos 10 y 15), la docente ayudaba a los estudiantes a reflexionar sobre las afirmaciones que estaban haciendo (turno14), y en el transcurso de la interacción los estudiantes lograron contrastar las ideas de las dos analogías (turnos 30 y 31) y comenzaron a confiar en la analogía creada por ellos, lo que resultó en su aceptación, ya que, asimismo, lograba representar claramente la dinamicidad propuesta en el átomo de Thomson.

En este sentido, notamos que, como lo destacó Sasseron (2018), debido a que se trata de un ambiente social de aprendizaje, el docente usó su autoridad epistémica para favorecer el desarrollo de la autonomía de los estudiantes, ya que estaba abierto a escuchar las ideas de los estudiantes y los cuestionaba con el fin de reflexionar sobre esas ideas.

Es importante resaltar que, según lo afirman Christodoulou y Osborne (2011), la forma en que el docente brinda a los estudiantes la oportunidad de involucrarse en el discurso en el aula, transfiriéndoles la responsabilidad de las problemáticas, favorece el desarrollo de la autonomía y su implicación en la discusión. Durante la interacción argumentativa 1, es considerable que los estudiantes, por más que estuvieran en conflicto con sus ideas, buscaban encontrar la mejor comparación para representar las ideas del átomo y con eso se involucraron en interacciones destinadas a la comunicación y proposición de ideas (turnos 4 y 5), críticas (turno 30) y justificaciones para las declaraciones de sus compañeros (turno 27). De esta manera, observamos que el contexto de resolución de problemas por parte de los estudiantes también puede favorecer el desarrollo de su responsabilidad de aprender, ya que se sienten comprometidos a encontrar la mejor solución.

Además, como lo resaltan Ferraz y Sasseron (2018), cuando el docente usa diferentes propósitos para interrogar a los estudiantes, como retomar la idea (turno 21) discutida en situaciones anteriores, explotar distintos puntos de vista (turno 6) y sintetizar las ideas (turno 36), esto puede contribuir con la implicación de los estudiantes en la discusión. Es importante tener en cuenta que la profesora intentó evaluar las ideas que surgieron en el ámbito social (turno 15) no como correctas o incorrectas, sino en busca de la reflexión de los estudiantes sobre esas ideas.

Notamos que cuando la profesora usó su discurso para legitimar una idea presentada por el estudiante (turno 29), él posteriormente critica (turno 30) una característica del análogo "pudín de pasas" que estaría en contra de sus ideas sobre el átomo. Según Kelly y Licona (2018), cuando el docente utiliza la práctica del metadiscurso, que consiste en un proceso de legitimación del discurso del estudiante, puede brindar oportunidades para la participación e implicación de los estudiantes con las prácticas epistémicas. En este caso, observamos que el apoyo de la docente favoreció la implicación del estudiante y el desarrollo de la autonomía para criticar la información del libro de texto.

Observamos que, aunque los estudiantes no desarrollaron autonomía para involucrarse en interacciones interpersonales con sus compañeros, a medida que el docente realizaba el 
movimiento de escucha, proponían diferentes ideas (turnos 4 y 16) y completaban las que surgían en el ámbito social (turnos 5 y 17), no dejando este rol exclusivamente al docente.

La participación de los estudiantes durante la interacción argumentativa transcrita en la tabla 3 también revela que hay receptividad a la crítica, ya que la evaluación de ideas por parte de la docente (turnos 15 y 18) no la sienten como un punto final de la discusión, sino que se produce como un paso de profundización y reflexión sobre las propuestas de los estudiantes (turnos 14 y 16), lo que permite, incluso, que las ideas se discutan a partir de la reanudación de ideas ya discutidas en otros momentos (turnos 21 y 25). Ferraz y Sasseron (2017) denominan momentos como este un "espacio interactivo para la argumentación colaborativa": un espacio en el que, a partir de las discusiones, se establecen puntos de vista y se los evalúa a la luz de las evidencias y el conocimiento ya construido.

Además, notamos que en la interacción argumentativa 1 hay igualdad moderada entre los miembros del aula: estudiantes y docente. La igualdad moderada es una de las normas para la construcción social del conocimiento en la ciencia, según Longino (2002). Para esta autora, esta es una de las normas que deben guiar la construcción del conocimiento, pues permite la discusión de ideas de modo que se considere que todos los participantes tienen autoridad intelectual, es decir, que son igualmente capaces de contribuir, en una relación más horizontal de poder. En nuestros datos, observamos que esta norma manejaba la interacción en el contexto del aula investigada, ya que la profesora considera las propuestas de los estudiantes (turno 15) y alienta la presentación de ideas por parte de ellos (turno 1, 21), siendo estas contribuciones consideradas y utilizadas (turno 36) para la construcción de una explicación común.

Después del momento de la selección de las analogías por parte de los grupos entre la analogía creada por los estudiantes y la del "pudín de pasas", la docente pidió a todos los grupos de la clase que fueran a la pizarra para presentar la analogía defendida por el grupo y para discutir sobre cuál de esos análogos expuestos sería el mejor para compararlo con el modelo atómico de Thomson. A continuación, en la tabla 4, presentamos la interacción argumentativa entre los estudiantes durante la elección del análogo, en discusión con toda la clase. En la transcripción, para asociar los miembros con el grupo al que pertenecía, pusimos los códigos G1, G2 y G3 (grupo 1, grupo 2 y grupo 3, respectivamente) seguidos del nombre del estudiante. El grupo de estudiantes analizado en este trabajo se identifica como "G1" en la tabla 4.

Tabla 4. Interacción argumentativa 2

\begin{tabular}{|c|l|l|}
\hline $\begin{array}{c}\text { Turnos } \\
\text { de habla }\end{array}$ & \multicolumn{1}{|c|}{ Transcripción } & \multicolumn{1}{c|}{ Aclaraciones } \\
\hline 1 & [CaioG1]: iEl nuestro! & $\begin{array}{l}\text { El grupo está a favor de la comparación con la } \\
\text { rueda del automóvil. }\end{array}$ \\
\hline 2 & {$[$ Profesora]: ¿Por qué? } & $\begin{array}{l}\text { La profesora solicita justificación para el } \\
\text { posicionamiento de los estudiantes. }\end{array}$ \\
\hline 3 & {$[$ AnaG3]: iEl pudín! } & $\begin{array}{l}\text { El grupo está a favor de la analogía con el “pudín } \\
\text { de pasas". }\end{array}$ \\
\hline 5 & Profesora]: ¿Por qué? A ver. Uno por vez... & $\begin{array}{l}\text { La profesora cuestiona el motivo del } \\
\text { posicionamiento de los estudiantes. }\end{array}$ \\
\hline 5 & $\begin{array}{l}{[\text { AnaG3]: Porque ya tiene las cosas que representan }} \\
\text { los electrones... }\end{array}$ & $\begin{array}{l}\text { El grupo justifica su posición a favor de la } \\
\text { analogía con el “pudín de pasas" porque las pasas } \\
\text { representan los electrones. }\end{array}$ \\
\hline
\end{tabular}

Tabla 4. Continuación 


\begin{tabular}{|c|c|c|}
\hline $\begin{array}{l}\text { Turnos } \\
\text { de habla }\end{array}$ & Transcripción & Aclaraciones \\
\hline 6 & $\begin{array}{l}\text { [Profesora]: Los chicos piensan que es el pudin, } \\
\text { miren, porque los electrones se distribuirían, así } \\
\text { como las pasas en el pudin... ¿Están de acuerdo? }\end{array}$ & $\begin{array}{l}\text { La profesora reelabora la justificación de los } \\
\text { estudiantes y expone la idea del grupo a la clase. }\end{array}$ \\
\hline 7 & [ArthurG1]: Pero no, no se mueve... & $\begin{array}{l}\text { El grupo } 1 \text { critica el posicionamiento del grupo } 3 \\
\text { porque las pasas no representan el movimiento de } \\
\text { los electrones. }\end{array}$ \\
\hline 8 & [Profesora]: ¿Están de acuerdo? & $\begin{array}{l}\text { La profesora solicita la participación de los } \\
\text { estudiantes. }\end{array}$ \\
\hline 9 & [CaioG1]: No. & \multirow{2}{*}{ Los grupos están en desacuerdo sin justificación. } \\
\hline 10 & [BrunaG3]: No. & \\
\hline 11 & [Profesora]: ¿Por qué? & La profesora solicita justificación. \\
\hline 12 & $\begin{array}{l}\text { [ArthurG1]: Creo que no se mueven, no... Porque } \\
\text { los electrones están en constante movimiento, y las } \\
\text { pasas no se mueven dentro del pudin... }\end{array}$ & $\begin{array}{l}\text { El grupo argumenta con el objetivo de evaluar la } \\
\text { relación entre los electrones y las pasas propuesta } \\
\text { en la analogía con el "pudín de pasas". }\end{array}$ \\
\hline 13 & $\begin{array}{l}\text { Profesora]: A ver. Los chicos aqui dicen que no } \\
\text { están de acuerdo. }\end{array}$ & $\begin{array}{l}\text { La profesora problematiza la elección para toda la } \\
\text { clase a partir del argumento del grupo } 1 .\end{array}$ \\
\hline 14 & [AnaG3]: Pero en el nuestro están neutros... & $\begin{array}{l}\text { El grupo contrargumenta en defensa de la } \\
\text { analogía con el "pudín de pasas" afirmando que } \\
\text { en los átomos neutros los electrones son } \\
\text { estáticos, como las pasas en el pudín. }\end{array}$ \\
\hline 15 & $\begin{array}{l}\text { [Profesora]: Ok. Pero incluso el átomo neutro. } \\
\text { ¿Los electrones se mueven o no? }\end{array}$ & $\begin{array}{l}\text { La profesora busca reflexionar sobre la } \\
\text { declaración del grupo } 3 .\end{array}$ \\
\hline 16 & [AnaG3]: ;No! & $\begin{array}{l}\text { El grupo confirma la idea de la estabilidad del } \\
\text { átomo neutro. }\end{array}$ \\
\hline 17 & [CarlosG1]: Se mueven. & $\begin{array}{l}\text { El grupo no está de acuerdo con la idea de un } \\
\text { átomo estático. }\end{array}$ \\
\hline 18 & $\begin{array}{l}\text { [Profesora]: Se mueve. Los electrones están en } \\
\text { constante movimiento alli en los anillos. }\end{array}$ & $\begin{array}{l}\text { La profesora legitima la idea de un átomo } \\
\text { permanentemente dinámico, con anillos de } \\
\text { electrones en movimiento. }\end{array}$ \\
\hline 19 & [AnaG3]: Entonces es el de ellos... & $\begin{array}{l}\text { El grupo cambia su opinión y legitima la analogía } \\
\text { con la rueda del automóvil debido a la } \\
\text { dinamicidad propuesta en esa analogía. }\end{array}$ \\
\hline 20 & $\begin{array}{l}\text { [CarlosG1]: ¡Oh! Es el nuestro [refiriéndose a } \\
\text { la comparación que crearon], la pelota de } \\
\text { fútbol está parada... }\end{array}$ & $\begin{array}{l}\text { El grupo legitima la comparación con la rueda del } \\
\text { automóvil creada por ellos y evalúa la } \\
\text { comparación de la pelota de fútbol sugerida por } \\
\text { otro grupo de la clase. }\end{array}$ \\
\hline 21 & $\begin{array}{l}\text { [ArthurG1]: La pelota que rebota }{ }^{6} \text { también está } \\
\text { detenida... }\end{array}$ & $\begin{array}{l}\text { El grupo evalúa la comparación con la pelotita } \\
\text { sugerida por otro grupo de la clase porque no } \\
\text { representa la dinamicidad del átomo. }\end{array}$ \\
\hline 22 & [JoãoG1]: ¡Es la nuestra! & $\begin{array}{l}\text { El grupo afirma que la comparación sugerida por } \\
\text { ellos (rueda del automóvil) es mejor que las } \\
\text { demás para representar el átomo. }\end{array}$ \\
\hline 23 & $\begin{array}{l}\text { [ArthurG1]: El cö̈nete también rueda solo por } \\
\text { dentro, pero si dejas de girarlo también se detiene... } \\
\text { En el nuestro no, el movimiento de la rueda puede } \\
\text { detenerse que en su interior seguirá girando. }\end{array}$ & $\begin{array}{l}\text { Legitima la comparación del grupo y evalúa la } \\
\text { comparación con el cojinete sugerido por el otro } \\
\text { grupo de la clase con la analogía que ellos mismos } \\
\text { crearon con la rueda del automóvil. }\end{array}$ \\
\hline
\end{tabular}

\footnotetext{
${ }^{6}$ Este tipo de pelotita se libera en máquinas que funcionan a partir de la introducción de monedas. Estas pelotitas tienen diferentes puntos de colores dispersos en su interior y los estudiantes sugirieron comparar la mezcla de colores con los electrones en el átomo.
} 
A partir de la interacción argumentativa 2 descrita en la tabla 4, podemos ver que los estudiantes discutieron diferentes comparaciones. Con el objetivo de que quede más claro para el lector, a continuación, expondremos las relaciones establecidas en cada una de ellas a partir de las tablas 5, 6 y 7. La primera analogía citada por los estudiantes (turno 1) es la existente entre el modelo atómico de Thomson y la rueda del automóvil, creada por los estudiantes del grupo 1, y las relaciones de esta analogía también se explotaron previamente en la tabla 3. La segunda analogía citada por los estudiantes (turno 3) se refiere a la que existe entre el modelo atómico de Thomson y el "pudín de pasas", las relaciones de esta comparación se explotaron anteriormente en la tabla 2. Las comparaciones con el cojinete, la pelotita que rebota y la pelota de fútbol se muestran en las tablas 5,6 y 7, respectivamente.

Tabla 5. Representación de las correspondencias de la comparación con el cojinete

\begin{tabular}{|c|c|c|}
\hline $\begin{array}{c}\text { Cojinete } \\
\text { (análogo) }\end{array}$ & Correspondencias & $\begin{array}{c}\text { Modelo atómico de Thomson } \\
\text { (objetivo) }\end{array}$ \\
\hline $\begin{array}{c}\text { Movimiento de las esferas en el } \\
\text { sistema de cojinete }\end{array}$ & & $\begin{array}{c}\text { Movimiento de los electrones en } \\
\text { el átomo }\end{array}$ \\
\hline
\end{tabular}

Tabla 6. Representación de las correspondencias de la comparación con la pelotita que rebota

\begin{tabular}{|c|c|c|}
\hline $\begin{array}{c}\text { Pelotita "que rebota" } \\
\text { (análogo) }\end{array}$ & Correspondencias & $\begin{array}{c}\text { Modelo atómico de Thomson } \\
\text { (objetivo) }\end{array}$ \\
\hline Forma esférica de la pelotita & Forma esférica del átomo \\
\hline $\begin{array}{c}\text { Elementos coloridos presentes } \\
\text { dentro de la esfera }\end{array}$ & $-\mathbf{I}$ | I I I I I I I & $\begin{array}{c}\text { Electrones presentes dentro de la } \\
\text { esfera }\end{array}$ \\
\hline
\end{tabular}

Tabla 7. Representación de las correspondencias de la comparación con la pelota de fútbol

\begin{tabular}{|c|c|c|}
\hline $\begin{array}{c}\text { Pelota de fútbol } \\
\text { (análogo) }\end{array}$ & Correspondencias & $\begin{array}{c}\text { Modelo atómico de Thomson } \\
\text { (objetivo) }\end{array}$ \\
\hline Forma esférica de la pelota & - I I I I I I I| I. & Forma esférica del átomo \\
\hline
\end{tabular}

Durante la interacción argumentativa 2 (tabla 4) notamos que los estudiantes del grupo 1 estaban convencidos de que la analogía que ellos mismos habían creado era mejor que todas las demás desde el comienzo de la interacción (turno 2). Por lo tanto, observamos que la interacción argumentativa 1 (tabla 1) era importante para que esos estudiantes se sintieran convencidos del potencial de la analogía que habían creado. Esto se debe a que el argumento sobre la dinamicidad del átomo utilizado por ellos para defender la comparación con la rueda del automóvil se basó en la interacción argumentativa 1, en la cual, según la interacción de la docente, las ideas de los estudiantes fueron legitimadas.

Nuestros datos son coherentes con la afirmación de Chiaro y Leitão (2005), quien declara que son las interacciones del profesor las que confieren status epistémico a las ideas de los estudiantes, pues observamos que en la interacción argumentativa 1 estas interacciones legitimaban las ideas de los estudiantes. Esto favoreció el desarrollo de su autonomía, como se evidenció anteriormente. A partir de esto, en la interacción argumentativa 2, los estudiantes actuaron como agentes epistémicos evaluando (turno 23), argumentando (turno 12), criticando (turno 7) y legitimando (turno 20) las ideas de sus compañeros en el ámbito social del aula. Los estudiantes del grupo 1 demostraron autonomía para, incluso, contrastar la analogía que habían creado con todas las analogías propuestas por los demás grupos de la clase (turnos $20 \mathrm{a}$ 23). 
En el turno 23 observamos que los estudiantes reconocieron las lagunas en las explicaciones propuestas por otros compañeros, cuando evaluaron críticamente la comparación con el cojinete. Los estudiantes observaron que a pesar de que la comparación con el cojinete mostraba una relación con la dinamicidad del átomo, esta relación presentaba una laguna, ya que los electrones estaban en constante movimiento dentro del átomo y la analogía con el cojinete sugería que el movimiento de los electrones dependía del movimiento del exterior del átomo cuando pensaron en el funcionamiento del cojinete. Jiménez-Aleixandre et al. (2000) afirman que el reconocimiento de los estudiantes sobre las lagunas en las explicaciones que surgen en el ámbito social no es común en ambientes argumentativos. Creemos que esta es un fuerte indicador del desarrollo de la autonomía y responsabilidad de los estudiantes en este proceso, ya que reconocieron estas lagunas a la luz de las evidencias sin la necesidad de legitimación, por parte del docente, de esas ideas debatidas.

Consideramos que la actuación de los estudiantes como agentes epistémicos también se vio favorecida por el contexto del aula, que se configuró en una comunidad de práctica, en la que el profesor llevó a cabo acciones que alentaron la participación de los estudiantes en las prácticas discursivas, con sus propias ideas moldeando las discusiones Esto permitió la construcción de identidad por parte de los estudiantes, quienes asumieron responsabilidad y autonomía sobre el conocimiento, ya que sintieron que eran una parte activa de ese contexto.

\section{Consideraciones finales}

En el presente trabajo buscamos analizar cómo los estudiantes desarrollan autonomía y responsabilidad y actúan como agentes epistémicos en ambientes argumentativos a partir de la elección entre analogías.

El análisis realizado muestra que, en un principio, los estudiantes parecían no sentir seguridad para formular argumentos en defensa de sus proposiciones. En ese momento, las acciones del docente fueron fundamentales para legitimar las ideas de los estudiantes y apoyar el desarrollo de la autonomía por parte de ellos. La literatura (Kelly y Licona, 2018; Ferraz y Sasseron, 2017) señala que las acciones del profesor con el objetivo de explotar puntos de vista, resumir ideas discutidas en situaciones anteriores y legitimar las ideas de los estudiantes desde la práctica del metadiscurso son importantes para involucrarlos en la discusión en el aula.

Nuestros datos mostraron que estas acciones fueron importantes para que los estudiantes reflexionaran sobre sus propias ideas, se sintieran más seguros al expresarlas y, de esa forma, desarrollaran un cierto grado de autonomía en la evaluación crítica de la analogía presentada en el libro de texto y se implicaran en interacciones interpersonales con sus compañeros en un segundo momento.

Por lo tanto, en un segundo momento, cuando los estudiantes corroboraron sus proposiciones y se convencieron de su validez, se implicaron en interacciones interpersonales con sus compañeros en defensa de la analogía que ellos mismos habían creado. Autores del área (Jiménez-Aleixandre, 2000; Chiaro y Leitão, 2004) argumentan que no es común que los estudiantes encuentren lagunas en las explicaciones de sus compañeros ni que se involucren en interacciones para evaluar y criticar las ideas que surgen en la esfera social. Los datos de esta investigación muestran que, después del momento de interacción con el profesor, en el que él brindó un status epistémico (Chiaro y Leitão, 2004) a las ideas de los estudiantes, demostraron autoridad para criticar la analogía con el "pudín de pasas", lograron identificar lagunas en las comparaciones presentadas por sus compañeros, evaluaron las ideas que surgieron en el ámbito social y argumentaron en defensa de esas ideas personales. Al identificar esta relación entre la implicación de los estudiantes y la forma en que la docente condujo la discusión, notamos que nuestros resultados son congruentes con los del trabajo de Araújo y Mortimer 
(2009), que llaman la atención sobre el hecho de que la participación de los estudiantes en las actividades del aula de Química están directamente relacionadas con las estrategias utilizadas por el docente.

En línea con las notas de Kelly y Cunningham (2019), pudimos observar que el trabajo de los estudiantes con herramientas epistémicas que apoyan la construcción y negociación del conocimiento favoreció el desarrollo de su autonomía y responsabilidad. En el contexto investigado, las analogías autorales funcionaron como herramientas epistémicas, favoreciendo el desarrollo de la autonomía de los estudiantes al actuar en defensa de esas representaciones personales.

Notamos que el contexto de resolución de problemas también favoreció la responsabilidad del estudiante por su aprendizaje, en la medida en que se sintieron comprometidos a tomar una decisión bien fundamentada. Este resultado es consistente con el trabajo de Christodoulou y Osborne (2011), quienes afirman que, cuando el docente realiza acciones con el objetivo de transferir la responsabilidad de los temas discutidos en el aula a los estudiantes, esto favorece el desarrollo de su autonomía en la discusión. Aunado a esto, se subraya la naturaleza del problema. Mortimer y Araújo (2014) observaron que el uso de situaciones cotidianas para problematizar temas en las clases de Química ayuda a los estudiantes a involucrarse y desarrollar su autonomía y responsabilidad por ellos. En nuestro contexto, este acercamiento entre la problemática y la vida cotidiana de los estudiantes se produjo a través de la elección de las comparaciones, a partir de las cuales compararon el dominio objetivo (modelo atómico de Thomson) con uno análogo que les era familiar.

Además, observamos que la forma como se condujo la discusión favoreció un contexto de igualdad moderada entre los miembros del aula: estudiantes y docente. Según Longino (2002), esta igualdad moderada es una de las normas para la construcción social del conocimiento en la ciencia. En el contexto de enseñanza investigado, mostramos que favorecer esta igualdad permitió a los estudiantes autonomía para interactuar y colaborar con la discusión.

Cabe resaltar que, al evaluar el desarrollo de la agencia epistémica de los estudiantes en el contexto investigado, dirigimos nuestra atención a las interacciones argumentativas y a cómo el conocimiento fue puesto a disposición por la docente y articulado por los estudiantes en el proceso de construcción social en el aula. Sin embargo, somos conscientes de que las percepciones y la implicación de los estudiantes con los problemas abordados pueden verse afectados por sus experiencias personales dentro y fuera del contexto escolar (Ko y Krist, 2019; Miller, Manz, Russ, Stroupe, y Berland, 2018). Coincidimos con Ko y Krist (2019) en que aspectos afectivos como el entusiasmo, la animación y la frustración pueden influir en la implicación y la adhesión de los estudiantes en los procesos discursivos del aula. Sin embargo, como sugieren estos autores, las pruebas empíricas relacionadas con las experiencias afectivas de los estudiantes son necesarias para identificar las interferencias de estos aspectos en el aula. Consideramos que esta es una limitación de nuestro estudio, ya que no exploramos los aspectos emocionales y afectivos relacionados con el desarrollo de la agencia epistémica de los estudiantes. Finalmente, concluimos que cuatro aspectos fueron fundamentales para el desarrollo de la autoridad y responsabilidad de los estudiantes, haciéndolos actuar como agentes epistémicos en la enseñanza de Química, a saber: (i) la actuación de la docente con acciones dirigidas a apoyar la participación de los estudiantes en el discurso y la legitimación de sus ideas, (ii) la igualdad moderada permitida entre los miembros del aula, (iii) el contexto de resolución de problemas, que favoreció la responsabilidad de los estudiantes de buscar soluciones coherentes y fundamentadas y (iv) el trabajo con analogías autorales como herramientas epistémicas. 


\section{Agradecimientos}

Los autores agradecen al Consejo Nacional de Desarrollo Científico (CNPq) y Tecnológico, la Coordinación para el Perfeccionamiento del Personal de Educación Superior, Brasil (CAPES) el Código de Financiamiento 001.

\section{Referencias}

Aragón, M. M., y Oliva, J. M. (2013). Evolución de los modelos explicativos de los alumnos en torno al cambio químico a través de una propuesta didáctica con analogias. Enseñanza de las Ciencias, 31(2), 9-30.

Araújo, A. O., y Mortimer, E. F. (2009). As práticas epistêmicas e suas relações com os tipos de texto que circulam em aulas práticas de Quimica Paper presented at the VII Enpec (Encontro Nacional de Pesquisa em Educação em Ciências), Florianópolis, Santa Catarina, Brasil.

Baker, M. (2009). Argumentative Interactions and the Social Construction of Knowledge. In N. M. Mirza y A.-N. Perret-Clermont (Eds.), Argumentation and Education: Theoretical Foundations and Practices (pp. 127-144). Dordrecht: Springer.

Bernardino, M. A. D., Rodrigues, M. A., y Bellini, L. M. (2013). Análise crítica das analogias do livro didático público de química no estado do Paraná. Ciência y Educação, 19(1), 135150.

Blanco, J. L., Alemany, F. S., y Torregrosa, J. M. (2013). ¿Los Libros de Texto de Bachillerato Introducen Adecuadamente los Modelos Atómicos de Thomson y Rutherford? Enseñanza de las Ciencias 31(1), 29-43.

Botelho, K. F., y Pietrocola, M. (2017). A pesquisa baseada em design: visão geral e contribuições para o ensino de ciências. Investigações em Ensino de Ciências, 22(2).

Cascales, J. R., Blanco, G. S., y Pérez, M. V. (2018). Percepción de profesores y estudiantes de $3^{\circ}$ ESO sobre el uso de analogías en el estudio de los estados de agregación de la materia. Revista Eureka sobre Enseñanza y Divulgación de las Ciencias, 15(2), 1-15.

Chiaro, S., y Leitão, S. (2005). O papel do professor na construção discursiva da argumentação em sala de aula. Psicologia: Reflexão e crítica, 18(3), 350-357.

Christodoulou, A., y Osborne, J. (2014). The Science Classroomas a Site of EpistemicTalk: ACase Study of aTeacher's Attempts toTeach Science Based on Argument. Journal of Research in Science Teaching, 51(10), 1275-1300.

Cohen, L., Manion, L., y Morrison, K. (2011). Research Methods in Education (7 ed.). London and New York: RoutledgeFalmer.

Collins, A., Joseph, D., y Bielaczyc, K. (2004). Design research: Theoretical and methodological issues. The Journal of the learning sciences, 13(1), 15-42.

Crujeiras, B. (2014). Competências e Prácticas Cientificas no Laboratorio de Química: Participación do alumnado de secundaria na indagación. (Tese de Doutorado), Universidade de Santiago de Compostela, Santiago de Compostela.

Duit, R. (1991). On the role of analogies and metaphors in learning science. Science Education, 75(6), 649-672.

Engle, R. A., y Conant, F. R. (2002). Guiding Principles for Fostering Productive Disciplinary Engagement: Explaining an Emergent Argument in a Community of Learners Classroom. Cognition and Instruction, 20(4), 399-483. 
Ferraz, A. T., y Sasseron, L. H. (2017). Propósitos epistêmicos para a promoção da argumentação em aulas investigativas. Investigações em Ensino de Ciências, 22(1), 42-60.

Gentner, D. (1989). The mechanisms of analogical learning. In S. Vosniadou y A. Ortony (Eds.), Similarity and Analogical Reasoning (pp. 199-241). Cambridge: Cambridge University Press.

Glynn, S. M. (2007). Methods and strategies: Teaching with analogies. Science and Children, 44(8), 52-55.

Jiménez-Aleixandre, M. (2010). 10 ideas clave: competencias en argumentación y uso de pruebas. Barcelona: Graó.

Jiménez-Aleixandre, M., y Crujeiras, B. (2017). Epistemic pratices and scientific practices in science education. Science Education, 69-89.

Jiménez-Aleixandre, M., Bugallo Rodríguez, A., y Duschl, R. A. (2000). "Doing the Lesson" or "Doing Science": Argument in High School Genetics. Science Education, 84(6), 757-792.

Justi, R. (2006). La enseñanza de ciencias basada en la elaboración de modelos. Enseñanz̧a de las Ciencias, 24(2), 173-184.

Kelly, G. J. (2008). Inquiry, activity and epistemic practice. In R. Duschl y R. E. Grandy (Eds.), Teaching Scientific Inquiry: recommendations for research and implementation (pp. 288291). Rotterdam, Holand: Taipei Sense Publishers.

Kelly, G. J., y Cunningham, C. M. (2019). Epistemic tools in engineering design for K-12 education. Science Education 103(4), 1-32.

Kelly, G. J., y Duschl, R. A. (2002). Toward a research agenda for epistemological studies in science education. Paper presented at the Annual meeting of the National Association for Research in Science Teaching, New Orleans, LA.

Kelly, G., y Licona, P. (2018). Epistemic Practices and Science Education. In M. R. Matthews (Ed.), History, Philosophy and Science Teaching (pp. 139-165). Sydney, Australia: Springer.

Ko, M. L., y Krist, C. (2019). Opening up curricula to redistribute epistemic agency: A framework for supporting science teaching. Science Education, 103(4), 979-1010.

Lave, J. (1996). Teaching, as learning, in practice. Mind, culture, and activity, 3(3), 149-164.

Lave, J., y Wenger, E. (1991). Situated learning: Legitimate peripheral participation. New York: Cambridge University Press.

Longino, H. E. (2002). The fate of knowledge. Princeton: Princeton University Press.

Lopes, C. V., y Marques, D. M. (2010). Modelos atômicos de J. J. Thomson e Ernest Rutherford. In M. H. R. Beltran, F. Saito, y L. S. P. Trindade (Eds.), História da ciência: tópicos atuais 2 (pp. 131-158). São Paulo: Livraria da Física.

Lüdke, M., y André, M. E. D. A. (2015). Pesquisa em Educaşão: Abordagens qualitativas (2 ed.). Rio de Janeiro: E.P.U.

McNeill, K. L., María González-Howard, R. K., y Suzanna, L. (2017). Moving Beyond Pseudoargumentation: Teachers' Enactments of an Educative Science Curriculum Focused on Argumentation. Science Education, 101(3), pp. 426-457.

Miller, E. M., Russ, R., Stroupe, D., y Berland, L. K. (2018). Addressing the epistemic elephant in the room: Epistemic agency and the next generation science standards. Journal of Research in Science Teaching,, 55(7), 1053-1075. 
Mortimer, E. F., y Araújo, A. O. (2014). Using productive disciplinary engagement and epistemic practices to evaluate a traditional Brazilian high school chemistry classroom. International Journal of Educational Research, 64, 156-169.

Mozzer, N. B., y Justi, R. (2015). "Nem tudo que reluz é ouro": Uma discussão sobre analogias e outras similaridades e recursos utilizados no ensino de Ciências. Revista Brasileira de Pesquisa em Educação em Ciências, 15(1), 123-147.

Nascimento, L. A., y Sasseron, L. H. (2019). A constituição de normas e práticas culturais nas aulas de ciências: proposição e aplicação de uma ferramenta de análise. Revista Ensaio, 21, 1-22.

Osborne, J. (2016). Defining a knowledge base for reasoning in science: The role of procedural and epistemic knowledge. In R. A. DUSHL y A. S. BISMARCK (Eds.), Reconceptualizing STEAM Education: the central role of practice (pp. 215-231). New York: Routledge.

Osborne, J., MacPherson, A., Patterson, A., y Szu, E. (2012). Introduction. In M. S. Khine (Ed.), Perspectives on scientific argumentation (pp. 3-16). New York: London

Ramos, T. C., y Mozzer, N. B. (2018). Análise do Uso da Analogia com o "Pudim de Passas" Guiado pelo TWA no Ensino do Modelo Atômico de Thomson: considerações e recomendações. Quimica Nova na Escola, 40(2), 106-115.

Ramos, T. C., Mendonça, P. C. y Mozzer, N. B (2016). Argumentação na Elaboração e Crítica de Analogias: Unidade Didática para o Ensino dos Modelos Atômicos. Paper presented at the XVIII Encontro Nacional de Ensino de Química, Florianópolis, SC.

Rosa, C. T., Cótica, R. P., y Pereira, L. H. (2016). Analogias no estudo de eletricidade nos livros didáticos de física. Revista Electrónica de Enseñanza de las Ciencias, 15(3), 363-379.

Sasseron, L. H. (2018). Práticas em aula de ciências: o estabelecimento de interações discursivas no ensino por investigação. (Livre docência ), Universidade de São Paulo, São Paulo.

Sasseron, L. H., y Duschl, R. A. (2016). Ensino de Ciências e as Práticas Epistêmicas: O papel do professor e o engajamento dos estudantes. Investigações em Ensino de Ciências, 21(2), 52-67.

Silva, M. B., Gerolin, E. C., y Trivelato, S. L. F. (2018). A Importância da Autonomia dos Estudantes para a Ocorrência de Práticas Epistêmicas no Ensino por Investigação. Revista Brasileira de Pesquisa em Educação em Ciências, 18(3), 905-933.

Stroupe, D. (2014). Examining Classroom Science Practice Communities: How Teachers and Students Negotiate Epistemic Agency and Learn Science-as-Practice. Science Education, 98(3), 487-516.

Thiele, R. B., y Treagust, D. F. (1994). An interpretative examination of high school chemistry teachers' analogical explanations. Journal of Research in Science Teaching, 31(3), 227-242. 